\title{
Radiographic Analysis of the Lumbosacral Juncture: Is There a Critical Sacral Angle for Total Disc Replacement?
}

\author{
Rattalerk Arunakul ${ }^{1,2}$, Melodie Metzger $^{2}$, Linda Kanim² ${ }^{2}$, Hyun Bae $^{2}$, Michael Kropf $^{2}$, Rick Delamarter $^{2}$ \\ ${ }^{1}$ Department of Orthopaedics, Faculty of Medicine, Thammasat University, Pathumthani, Thailand \\ ${ }^{2}$ Spine Center, Cedars-Sinai Medical Center, Los Angeles, CA, USA
}

Study Design: Retrospective review of a patient cohort through a prospective study.

Purpose: To determine whether there are correlations between radiographic measurements, including sacral slope (SS) and pelvic incidence (PI), and self-reported clinical outcomes among single-level L5/S1 ProDisc-L patients.

Overview of Literature: The lumbosacral juncture presents unique biomechanical challenges with respect to artificial disc replacement (ADR) because of its orientation and consequential shear loading. Reports of inferior outcomes at L5/S1 compared to those of the outcomes at the levels above, including increased facet joint pain, suggest a relationship with the sacral inclination at L5/S1.

Methods: Plain standing lateral radiographs of 71 patients (age, 26-65 years) who underwent ADR at L5/S1 for degenerative disc disease were reviewed. SS and PI were measured based on pretreatment and initial follow-up standing films. Patient's average adjusted self assessments included the Oswestry disability index and visual analog scale for pain 2 years after ADR. Correlation coefficients were computed to evaluate relationships between radiographic parameters and clinical outcomes. Analysis of covariance was used to evaluate multivariate relationships among factors, including radiographic parameters, body mass index (BMI), and clinical outcomes. Results: SS and PI values were obtained from 71 patients. The average SS was $33.3^{\circ}$ and average PI was $39.9^{\circ}$. At the 24 -month follow-up, no significant correlations $(p \geq 0.05)$ were observed between radiographic parameters and clinical outcomes. BMI, age, and sex did not explain any variability in the relationships between clinical outcomes and SS and PI.

Conclusions: We reviewed a large range of SS angles and found no associations between SS, Pl, or BMl and clinical outcomes after $A D R$ at $L 5 / S 1$. These preliminary results demonstrate that ADR provided maintainence of pain relief and functional improvement for a wide range of SS angles, suggesting that steeper angles are not a contraindication for ADR.

Keywords: Lumbosacral slope; Total disc replacement; Clinical impact

\section{Introduction}

For many years, the lumbosacral junction has been recognized as an anatomically and mechanically challenging area of the spine [1]. Its oblique orientation and caudal location exposes it to extreme levels of compressive and shear forces that are not observed anywhere else in the spine. These difficulties are further complicated by the

\footnotetext{
Received Apr 8, 2016; Revised Aug 12, 2016; Accepted Aug 23, 2016

Corresponding author: Rattalerk Arunakul

Department of Orthopaedics, Faculty of Medicine, Thammasat University,

95 Moo 8, Paholyotin Road Klong Luang, Pathumthani, 12120 Thailand

Tel: +66-29269775, Fax: 66-29269793 , E-mail: benz.md@gmail.com
} 
wide variety of curvatures and morphologies within the general population.

Historically, the development of the sacrovertebral angle and corresponding lumbar lordosis has been related to the progressive acquisition of an erect posture and the ontogeny of bipedal locomotion. This evolutionary process that distinguishes us from our ancestors has not been perfected and is continually changing to enable the comfortable maintenance of an erect posture. As a consequence, the sacrovertebral angle and lordotic curve exhibit a relatively broad frequency of distribution $[2,3]$.

Stability at the lumbosacral junction depends on the orientation of L5 with respect to the sacrum, lumbosacral angle, sacral slope (SS), and pelvic incidence (PI). Alterations in these spinopelvic parameters have been associated with a variety of pathological conditions, including low back pain [4,5], disc degeneration [6,7], and spondylolisthesis [8-10]. This suggests that morphological abnormalities play a role in the stresses and loads experienced by the disc, facets, and surrounding tissues that predispose a patient to spinal disorders. In 2005, Keller et al. [11] analyzed this relationship in a cross-sectional study and showed a correlation between lumbar shear and compressive stresses and T12-S1 sagittal balance, lumbar lordosis, and SS, confirming that the spinal column morphology dictates mechanical factors that, in turn, influence the frequency of lumbar spine pathologies.

Of particular interest is the inclination of the upper surface of the sacrum (S1) in relation to the horizon. The more marked this inclination, the greater the tendency of L5 to slip downward and forward, creating greater strain on the ligments, muscles, intervertebral disc, and articular processes. Previous studies have recognized the L5/S1 junction as a challenging region for arthrodesis [12,13], but to date, there have been no investigations on its influence on artificial disc replacement (ADR) procedures despite clinical reports of outcomes at L5/S1 being inferior to those at the levels above $[14,15]$. In addition, the facets, which are largely responsible for resisting the forward displacement of L5 on S1, have been reported to show increased levels of degeneration and arthritis post-ADR implantation [16,17].

Therefore, the purpose of this initial investigation was to determine whether there are relationships between radiographic measurements, including SS and PI, and selfreported clinical outcomes of visual analogue scale (VAS) back pain (VAS pain) and Oswestry disability index (ODI) among single-level L5/S1 ADR patients. Existing data from a clinical investigation were used to examine possible relationships that are interesting for further specifically designed studies.

\section{Materials and Methods}

\section{Design}

This study entailed an additional post-hoc analysis of existing data collected prospectively in the multicenter randomized clinical trial of ProDisc-L (Spine Solutions/ Synthes Spine, Solothurn, Switzerland) vs. $360^{\circ}$ Fusion. A retrospective detailed review of radiographs was completed and additional measurements were collected for a subcohort of patients with degenerative disc disease treated only at L5/S1 with a ProDisc-L. These additional parameters were analyzed against existing outcome data to generate working hypotheses on the relationship among SS, PI, and clinical outcomes after ProDisc-L.

\section{Radiographic measurements}

Plain standing lateral radiographs were reviewed and selected by an author (R.A.) for viewing the femoral head. SS and PI were measured on preoperative and/or initial follow-up standing sagittal radiographs. SS was determined as the angle between a horizontal line (parallel to the floor) and a parallel line on or with respect to the superior endplate of S1. PI was determined as the angle between a line drawn from the center of femoral head to the centroid of the superior endplate of $\mathrm{S} 1$ and a line perpendicular to the superior sacral vertebral endplate (Fig. 1).
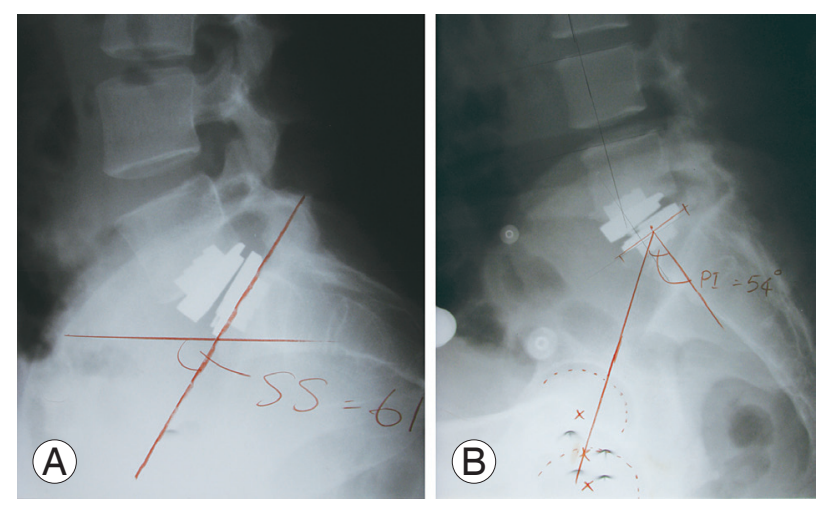

Fig. 1. (A) Sacral slope measurement. (B) Pelvic incidence measurement. SS, sacral slope; PI, pelvic incidence. 
Table 1. Descriptive information of patients and self-assessment outcomes

\begin{tabular}{lccccc} 
Variable & No. & Mean & Minimum & Maximum & Standard deviation \\
\hline Age $(\mathrm{yr})$ & 71 & 46.55 & 26.48 & 65.13 & 8.64 \\
BMI $\left(\mathrm{kg} / \mathrm{m}^{2}\right)$ & 71 & 25.69 & 18.17 & 35.63 & 3.78 \\
\hline Preoperative VAS & 71 & 6.93 & 1.3 & 9.8 & 1.98 \\
2-Year VAS & 63 & 2.98 & 0 & 9.81 & 2.61 \\
\hline Preoperative ODI & 71 & 29.76 & 19 & 40 & 5.66 \\
\hline 2-Year ODI & 64 & 13.86 & 0 & 38 & 1.07 \\
\hline
\end{tabular}

$\mathrm{BMI}$, body mass index; VAS, visual analogue scale; ODI, Oswestry disability index.

Table 2. Sacral slope (SS) and pelvic incidence (PI) data

\begin{tabular}{lccccc} 
Variable & No. & Mean & Minimum & Maximum & Standard deviation \\
SS & 55 & 33.25 & 22 & 61 & 7.62 \\
Low $\left(<35^{\circ}\right)$ & 31 & 28 & 22 & 34 & 3.67 \\
Medium $\left(35^{\circ}-45^{\circ}\right)$ & 20 & 37.90 & 35 & 42 & 2 \\
High $\left(>45^{\circ}\right)$ & 4 & 50.75 & 46 & 61 & 6.95 \\
$\mathrm{Pl}$ & 29 & 39.56 & 10 & 54 & 9.78 \\
\hline
\end{tabular}

\section{Patient self assessments and characteristics}

Patients previously completed a series of questionnaires as part of their normal follow-up and participation in the clinical trial. The self assessments included the ODI and VAS for back pain at 6 weeks and 3, 6, 12, and 24 months after surgery. The outcomes at 24 months were used as one of the dependent variables. For VAS back pain and ODI, a second type of outcome measure was computed as the average adjusted across all postoperative time intervals for each patient, yielding a stable outcome parameter as the average VAS ( $\mathrm{VVAS}_{\text {pain }}$ ) and average ODI (aODI) for each patient. Patient characterstics were collected through the standard intake questionnaires that were given as part of the initial screening and clinical trial work-up.

\section{Statistical analysis}

We compared the preoperative and postoperative selfassessment outcomes (VAS and ODI) between each group (SS and ODI) using the Paired $t$ test for normally distributed data and Wilcoxon signed-rank test for non-normally distributed data. Correlations between radiographic (SS and PI) and clinical self-assessment outcomes (VAS and ODI) were calculated using Pearson's correlation analysis for normal distribution and Spearman's correlation for non-normal distribution. Multivariate analysis of variance was also used to evaluate clinical outcomes as a function of the radiographic parameters SS and PI and other patient characteristics, including body mass index (BMI), patient age, and sex. All analyses were performed using Stata software ver. 14.0 (StataCorp., College Station, TX, USA).

\section{Results}

Seventy-one patients (age, 26-65 years) who underwent single-level ADR at L5/S1 for degenerative disc disease were reviewed. The SS and PI could be measured from radiographs in 55 and 29 of these patients, respectively. These 71 patients comprised the subcohort of this study; $56.3 \%(40 / 71)$ were males, the average age was 46 years (range, 26-64 years), and the average BMI was 25.6 (range, 18.1-35.6) (Table 1).

The average SS was $33.3^{\circ}\left(22^{\circ}-61^{\circ}\right)$ and average PI $\left(10^{\circ}-\right.$ $54^{\circ}$ ) was $39.6^{\circ}$ (normal range SS, $41^{\circ} \pm 8.4^{\circ}$; PI, $55^{\circ} \pm 9.8^{\circ}$ ) [18]. On the basis of the findings of an anatomical study in a normal population [19], we classified SS into 3 groups: low, $<35^{\circ}$; medium, $35^{\circ}-45^{\circ}$; and high, $>45^{\circ}$. The majority of SS data fell into the low range $\left(<35^{\circ}\right)$, and the average PI value also was low (Table 2).

Postoperative VAS for pain and ODI of the SS and PI 
Table 3. Preoperative and postoperative self-assessment outcomes comparison in SS and PI groups

\begin{tabular}{lrrr} 
Variable & Preoperative & 2-Year follow-up & $p$-value \\
SS & & & $<.001$ \\
VAS & $6.90 \pm 2.10$ & $3.11 \pm 2.63$ & $<0.001$ \\
\hline ODI & $30.00 \pm 5.73$ & $14.71 \pm 11.05$ & $<0.001$ \\
PI & & & $<.02 \pm 2.47$ \\
VAS & $6.80 \pm 2.05$ & $15.04 \pm 10.83$ & $<0.001$ \\
\hline ODI & $29.10 \pm 5.39$ & & \\
\hline
\end{tabular}

SS, sacral slope; PI, pelvic incidence; VAS, visual analogue scale; ODI, Oswestry disability index.

Table 4. Correlation between radiographic parameters and selfassessment outcomes

\begin{tabular}{|c|c|c|}
\hline Variables & $\begin{array}{l}\text { Correlation } \\
\text { coefficient (r) }\end{array}$ & $p$-value \\
\hline SS and Preop VAS & 0.33 & $0.01^{\mathrm{bl}}$ \\
\hline SS and 2-year VAS ${ }^{c)}$ & 0.05 & 0.75 \\
\hline SS and Preop ODI & 0.09 & 0.49 \\
\hline SS and 2-year ODI & 0.002 & 0.99 \\
\hline PI and Preop VAS ${ }^{a)}$ & 0.16 & 0.41 \\
\hline $\mathrm{PI}$ and 2-year VAS & -0.11 & 0.58 \\
\hline $\mathrm{PI}$ and Preop ODI & 0.26 & 0.17 \\
\hline $\mathrm{PI}$ and 2-year $\mathrm{ODI}{ }^{\mathrm{c}}$ & -0.17 & 0.39 \\
\hline
\end{tabular}

SS, sacral slope; Preop, preoperative; VAS, visual analogue scale; ODI, Oswestry disability index; PI, pelvic incidence.

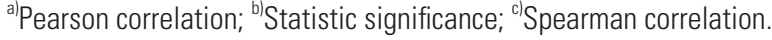

groups significantly improved (Table 3 ). Correlations among the patient's demographic data, radiographic parameters, and self-assessment clinical outcomes were evaluated. Sex and BMI were significantly correlated with age ( $r=-0.42, p=0.002 ; r=0.36, p=0.008$; resepectively). In terms of radiographic parameters, significant correlations were found between SS and sex $(r=-0.32, p=0.026)$ and between SS and preoperative VAS $(r=0.33, p=0.01)$ (Table 4). SS and PI were only marginally correlated $(r=0.39$, $p=0.07$ ) with each other, but interestingly, the correlation became statistically significant $(r=0.73, p=0.001)$ when BMI was included as a cofactor. At the 24-month followup, there were significant $(p<0.05)$ correlations among the self-assessment outcomes (preoperative VAS and ODI, average postoperative VAS and ODI, and average prepostoperative VAS and ODI) except for the preoperative and 2-year VAS ( $r=0.18, p=0.16)$. No significant correlations were observed between the average SS and ODI, SS and VAS, PI and ODI, and PI and VAS. There were also no significant correlations between SS and PI and any of the average adjusted measures (aODI and aVAS pain $)$. BMI did not explain any variability in the relationships between clinical outcome measures and SS and PI.

\section{Discussion}

PI and SS have been shown to be highly correlated with each other and with pelvic tilting, sagittal spinal curves, lordosis and kyphosis [20-22]. Roussouly et al. [23] described a wide variation in spinopelvic alignment and concluded that SS and pelvic alignment influence the lower lumbar lordotic curve. In addition, they determined that sagittal spinopelvic anatomy and alignment changes in severe spinal deformity play important roles in treating patients with spinal deformities. Wang et al. [24] showed that anatomical relationship patterns were altered in highgrade spondylolisthesis, especially for subjects with a retroverted sacropelvis. In these patients, correlations between PI and pelvic tilt and between the sacropelvic unit and lumbar spine geometry were lost [24]. Mac-Thiong et al. [25] studied spinopelvic alignment and its relevance to lumbosacral developmental spondylolisthesis. They concluded that posture is abnormal in high-grade spondylolisthesis associated with a retroverted sacropelvis, indicating that surgical reduction of the local lumbosacral deformity in these patients could be attempted to restore the normal posture [25]. Schwab et al. [18] found that pelvic tilt was highly correlated with patient self-reported function (ODI, SF-12, and scoliosis reserch society). They concluded that optimal treatment of patients with spinal deformity required integration of the pelvis in the preoperative evaluation and treatment plan.

Clearly, the relationship between spinal deformity and 
spinopelvic alignment has been recognized. Conversely, the correlation of spinopelvic alignment with surgical outcome of lumbar ADR has not been thoroughly explored. Theoretically, steep sacral inclination leads to increased shear stress on the implants when either ADR or fusion procedures are performed [11]. This might explain why some recent studies have shown outcomes of L5/S1 TDR being inferior to those of L4/5. Siepe et al. [15] followed 99 patients for an average of 25.8 months and found that results deteriorated when monosegmental ADR was performed at the lumbosacral junction, with a tendency toward statistical significance at the 24-month followup. Fluoroscopically guided spine infiltrations confirmed that the incidences of postoperative pain from posterior joint structures were $9.1 \%$ and $28.1 \%$ for L4/L5 and L5/S1 TDR [15]. Failure analysis study was performed by Siepe et al. [16] The same findings were observed with inferior outcome and a significantly higher incidence of posterior joint pain after ADR at L5/S1 (21.6\%) and bisegmental TDR at L4/5/S1 (33.3\%), respectively, when compared with those of L4/5 TDR (14.8\%). However, Sinigaglia et al. [26] found no difference between L4/5 and L5/S1 TDR for postoperative 36-Item Short Form Health Survey, ODI, and VAS for pain. Furthermore, replacement of the L4/ L5 disc had a higher risk of complications, the majority of which were approach or junctional pathology related, than that of L5/S1 [26].

In this study, we investigated whether spinopelvic anatomy, in particular, SS and PI, could be a predictor of surgical outcomes after ADR at the lumbosacral junction. Because of radiographic problems with viewing the femoral head, we only obtained PI values for 29 patients and SS values for 55 for patients in the dataset. The average SS was $33^{\circ}$, which is lower than that reported in the literature (normal range, 40-60) [27-29]. This asymmetric distribution may have been because of the small number of patients or unintended selection bias of patients by the surgeon. A significant correlation was shown between SS and preoperative VAS pain. However, we did not show any significant correlations between the alignment parameters and postoperative clinical outcomes. This might be explained by the effectiveness of ADR to eliminate pain and improve patients' quality of life after surgery, an idea supported by the significant statistical improvement in postoperative self-assessment outcomes in both the SS and PI groups.

There were a number of study limitations that should be considered. Our study was retrospective in nature, and we were only able to obtain precise measurements of SS and PI in a relatively small number of patients. These study characteristics may have affected the results, such as by causing a mismatch in the statistical significance between the preoperative and postoperative ODI score and VAS for pain. In addition, this study was limited to a 24-month follow-up, and the negative effects of a high SS or other sacropelvic parameters may not have had the time to become evident. The ODI and VAS scores are also inherently limited and may not have been able to detect early signs of degeneration based on mismatched kinematics. Radiographic measures, especially of the index-level facets, would probably provide more insight. There is also the possibility that SS only affects clinical outcomes of ADR when combined with other confounding factors such as obesity.

\section{Conclusions}

Previous studies have not investigated whether ADR is an appropriate treatment for patients with steep SS angles. We reviewed a large range of SS angles $\left(22^{\circ}-61^{\circ}\right)$ and found no associations between SS or PI and clinical outcomes after treatment with an artificial disc at L5/S1. In addition, there were no relationships among SS, BMI, and clinical outcomes. These preliminary results demonstrate that ADR can provide maintainence of pain relief and functional improvement for a wide range of SS angles, suggesting that steeper angles are not a contraindication for ADR. Further studies incorporating longer-term clinical results are necessary to provide more insight into this clinically important question. Therefore, we recommend standing film radiography as a routine preoperative evaluation, which will enable quantification of these spinopelvic parameters. With a more complete data set, we may find that SS and PI have important implications for the evaluation and treatment of ADR outcomes and/or other surgical interventions.

\section{Conflict of Interest}

No potential conflict of interest relevant to this article was reported.

\section{ORCID}

Rattalerk Arunakul: 0000-0003-3473-8436 
Melodie Metzger: 0000-0001-8892-7035

Linda Kanim: 0000-0001-9008-8103

Hyun Bae: 0000-0002-6386-344x

Michael Kropf: 0000-0001-7912-6308

Rick Delamarter: 0000-0002-2124-6769

\section{References}

1. Mitchell GA. The lumbosacral junction. J Bone Joint Surg 1934;16:233-54.

2. Abitbol MM. Evolution of the lumbosacral angle. Am J Phys Anthropol 1987;72:361-72.

3. Abitbol MM. Sacral curvature and supine posture. Am J Phys Anthropol 1989;80:379-89.

4. Steinberg EL, Luger E, Arbel R, Menachem A, Dekel $\mathrm{S}$. A comparative roentgenographic analysis of the lumbar spine in male army recruits with and without lower back pain. Clin Radiol 2003;58:985-9.

5. Harrison DD, Cailliet R, Janik TJ, Troyanovich SJ, Harrison DE, Holland B. Elliptical modeling of the sagittal lumbar lordosis and segmental rotation angles as a method to discriminate between normal and low back pain subjects. J Spinal Disord 1998;11:4309.

6. Kumar MN, Baklanov A, Chopin D. Correlation between sagittal plane changes and adjacent segment degeneration following lumbar spine fusion. Eur Spine J 2001;10:314-9.

7. Schlegel JD, Smith JA, Schleusener RL. Lumbar motion segment pathology adjacent to thoracolumbar, lumbar, and lumbosacral fusions. Spine (Phila Pa 1976) 1996;21:970-81.

8. Hosoe H, Ohmori K. Degenerative lumbosacral spondylolisthesis: possible factors which predispose the fifth lumbar vertebra to slip. J Bone Joint Surg Br 2008;90:356-9.

9. Hammerberg KW. New concepts on the pathogenesis and classification of spondylolisthesis. Spine (Phila Pa 1976) 2005;30(6 Suppl):S4-11.

10. Labelle H, Roussouly P, Berthonnaud E, Dimnet J, O'Brien M. The importance of spino-pelvic balance in L5-s1 developmental spondylolisthesis: a review of pertinent radiologic measurements. Spine (Phila Pa 1976) 2005;30:S27-34.

11. Keller TS, Colloca CJ, Harrison DE, Harrison DD, Janik TJ. Influence of spine morphology on intervertebral disc loads and stresses in asymptomatic adults: implications for the ideal spine. Spine J 2005;5:297309.

12. Kim SY, Maeng DH, Lee SH, Jang JS. Anterior lumbar interbody fusion for lumbosacral junction in steep sacral slope. J Spinal Disord Tech 2008;21:33-8.

13. Cunningham BW, Lewis SJ, Long J, Dmitriev AE, Linville DA, Bridwell KH. Biomechanical evaluation of lumbosacral reconstruction techniques for spondylolisthesis: an in vitro porcine model. Spine (Phila Pa 1976) 2002;27:2321-7.

14. Siepe CJ, Hitzl W, Meschede P, Sharma AK, Khattab $\mathrm{MF}$, Mayer MH. Interdependence between disc space height, range of motion and clinical outcome in total lumbar disc replacement. Spine (Phila Pa 1976) 2009; 34:904-16.

15. Siepe CJ, Mayer HM, Heinz-Leisenheimer M, Korge A. Total lumbar disc replacement: different results for different levels. Spine (Phila Pa 1976) 2007;32:78290.

16. Siepe CJ, Korge A, Grochulla F, Mehren C, Mayer HM. Analysis of post-operative pain patterns following total lumbar disc replacement: results from fluoroscopically guided spine infiltrations. Eur Spine J 2008; 17:44-56.

17. Park CK, Ryu KS, Jee WH. Degenerative changes of discs and facet joints in lumbar total disc replacement using ProDisc II: minimum two-year followup. Spine (Phila Pa 1976) 2008;33:1755-61.

18. Schwab F, Lafage V, Patel A, Farcy JP. Sagittal plane considerations and the pelvis in the adult patient. Spine (Phila Pa 1976) 2009;34:1828-33.

19. Siepe CJ, Tepass A, Hitzl W, et al. Dynamics of improvement following total lumbar disc replacement: is the outcome predictable? Spine (Phila Pa 1976) 2009;34:2579-86.

20. Vialle R, Levassor N, Rillardon L, Templier A, Skalli W, Guigui P. Radiographic analysis of the sagittal alignment and balance of the spine in asymptomatic subjects. J Bone Joint Surg Am 2005;87:260-7.

21. Boulay C, Tardieu C, Hecquet J, et al. Sagittal alignment of spine and pelvis regulated by pelvic incidence: standard values and prediction of lordosis. Eur Spine J 2006;15:415-22.

22. Legaye J, Duval-Beaupere G. Sagittal plane alignment of the spine and gravity: a radiological and clinical evaluation. Acta Orthop Belg 2005;71:213-20.

23. Roussouly P, Gollogly S, Berthonnaud E, Dimnet J. 
Classification of the normal variation in the sagittal alignment of the human lumbar spine and pelvis in the standing position. Spine (Phila Pa 1976) 2005;30: 346-53.

24. Wang Y, Qiu Y, Wang B, et al. Radiologic analysis of factors predicting the surgical reduction of lumbar spondylolisthesis. Zhonghua Wai Ke Za Zhi 2009; 47:289-92.

25. Mac-Thiong JM, Wang Z, de Guise JA, Labelle H. Postural model of sagittal spino-pelvic alignment and its relevance for lumbosacral developmental spondylolisthesis. Spine (Phila Pa 1976) 2008;33:2316-25.

26. Sinigaglia R, Bundy A, Costantini S, Nena U, Finocchiaro F, Monterumici DA. Comparison of singlelevel L4-L5 versus L5-S1 lumbar disc replacement: results and prognostic factors. Eur Spine J 2009;18 Suppl 1:52-63.

27. Bernhardt M, Bridwell KH. Segmental analysis of the sagittal plane alignment of the normal thoracic and lumbar spines and thoracolumbar junction. Spine (Phila Pa 1976) 1989;14:717-21.

28. Stagnara P, De Mauroy JC, Dran G, et al. Reciprocal angulation of vertebral bodies in a sagittal plane: approach to references for the evaluation of kyphosis and lordosis. Spine (Phila Pa 1976) 1982;7:335-42.

29. Jackson RP, McManus AC. Radiographic analysis of sagittal plane alignment and balance in standing volunteers and patients with low back pain matched for age, sex, and size: a prospective controlled clinical study. Spine (Phila Pa 1976) 1994;19:1611-8. 